\title{
Laparoscopic vs Open Drainage of Complex Pyogenic Liver Abscess
}

\author{
Mohamed M Mogahed ${ }^{1}$, Ashraf A Zytoon², Basem Eysa ${ }^{3}$, Mohamed Manaa $^{4}$, Wessam Abdellatif ${ }^{5}$
}

\begin{abstract}
Complex pyogenic liver abscess (CPLA) is a rare fatal disease if untreated. Complex pyogenic liver abscess is a multilocular abscess more than 5 $\mathrm{cm}$ in diameter. Pyogenic liver abscess (PLA) is mainly treated by percutaneous aspiration or drainage under antibiotic cover. Surgical drainage is indicated if interventional radiology fails, if ruptured, or if associated with biliary or intra-abdominal pathology. Laparoscopic drainage is a promising management option.

Aim: To evaluate the safety and efficacy of laparoscopic drainage as a management of complex pyogenic liver abscesses in comparison to open surgical drainage.

Materials and methods: Combined retrospective and prospective comparative study of 48 patients having complex PLA who were admitted to NHTMRI and managed by either laparoscopic drainage or open surgical drainage from January 2012 to January 2020 as regards results, complications, perioperative morbidity, mortality, and possible recurrence. Twenty-six patients were managed by open drainage, and 22 patients by laparoscopic drainage. Culture sensitivity of pus was done for all patients. Patients having small, solitary, and unilocular PLA that responded to antibiotic treatment or/and percutaneous drainage were excluded. All patients were subjected to full clinical assessment, laboratory investigations, ultrasonography, computed tomography, or magnetic resonance images for the abdomen and pelvis.

Results: Forty-eight patients having complex PLA with a median age of 54.5 years were managed by either laparoscopic drainage (22 patients) or open surgical drainage (26 patients). The operation time and hospital stay were less, and oral feeding was started earlier in laparoscopic group. Wound infection was higher in open drainage group. Abscess recurrence occurred once in laparoscopic group and once in open surgery group, and both were successfully treated with percutaneous drainage. One laparoscopic operation was converted to open.

Conclusion: Both laparoscopic and open surgical drainage of PLA are safe and effective. Laparoscopic drainage has less operative time, morbidity, and hospital stay; however, open drainage is considered the management of choice for patients with severe sepsis or failed percutaneous drainage. Keywords: Laparoscopy, Open drainage, Pyogenic liver abscess.

World Journal of Laparoscopic Surgery (2020): 10.5005/jp-journals-10033-1395
\end{abstract}

\section{INTRODUCTION}

Complex pyogenic liver abscess (CPLA) is a rare potentially fatal condition if untreated. Complex pyogenic liver abscess is an abscess that is multilocular and more than $5 \mathrm{~cm}$ in diameter. Pyogenic liver abscess (PLA) is mainly treated by percutaneous aspiration or drainage under antibiotic cover. Surgical drainage is indicated if interventional radiology fails, if ruptured, or if associated with biliary or intra-abdominal pathology. In CPLA, percutaneous drainage may help to optimize clinical condition before surgery. ${ }^{1,2}$ Laparoscopic drainage is a promising surgical option. ${ }^{3}$

Large pyogenic multilocular abscesses usually need drainage, in addition to antibiotics for effective management. ${ }^{1}$ Antibiotics alone does not work because of large bacterial load, antibiotics inactivation, and ineffective medium for bacterial elimination. Effective drainage shortens the antibiotic therapy duration. The methods include percutaneous needle aspiration (PNA), percutaneous catheter drainage (PCD), open surgical drainage (OSD), and laparoscopic drainage (LD). ${ }^{4}$

Patients with small, solitary, and unilocular abscesses are best managed with percutaneous aspiration plus antibiotics, especially the young healthy patients. Debilitated patients, elderly, diabetic patients, and patients with multiple or CPLA have a higher failure rate with percutaneous aspiration. ${ }^{5}$

\section{Aim}

To evaluate the safety and efficacy of laparoscopic drainage as a management of complex pyogenic liver abscesses in comparison to open surgical drainage.
${ }^{1}$ Department of Surgery, National Hepatology and Tropical Medicine Research Institute, Cairo, Egypt

${ }^{2}$ Department of Radiodiagnosis, Faculty of Medicine, Menoufia University, Menoufia, Egypt

${ }^{3,4}$ Department of Tropical Medicine, National Hepatology and Tropical Medicine Research Institute, Cairo, Egypt

${ }^{5}$ Department of Radiology, National Hepatology and Tropical Medicine Research Institute, Cairo, Egypt

Corresponding Author: Ashraf A Zytoon, Department of Radiodiagnosis, Faculty of Medicine, Menoufia University, Menoufia, Egypt, Phone: +201000219818, e-mail: ashradio@gmail.com

How to cite this article: Mogahed MM, Zytoon AA, Eysa B, et al. Laparoscopic vs Open Drainage of Complex Pyogenic Liver Abscess. World J Lap Surg 2020;13(1):11-15.

Source of support: Nil

Conflict of interest: None

\section{Materials and Methods}

Combined retrospective and prospective comparative study was conducted in NHTMRI from January 2012 to January 2020 on 48 patients ( 20 males and 28 females) with a median age of 54.5 years (ranges between 34 years and 65 years) having complex liver abscesses managed by either laparoscopic drainage or open surgical drainage. The comparison is as regards results, 
complications, perioperative morbidity, mortality, and possible recurrence. Twenty-two patients were managed by laparoscopic drainage and 26 patients by open surgical drainage.

All patients were subjected to full clinical assessment, laboratory investigations ( $C B C, F B S, P P, H b A 1 C$, creatinine, liver enzymes, albumin and bilirubin levels, PT, PC, and INR), and at least one or two radiological investigations (ultrasonography, computed tomography, or magnetic resonance images for the abdomen and pelvis). Abdominal ultrasonography was done in all patients and computed tomography was done in 22 patients with well-defined lowattenuation lesion that is having enhancing peripheral rim with single multiloculated cystic appearance, and MRI was done in 2 patients with imaging feature of multiloculated cystic lesion of low T1 and high T2 signal with enhancing peripheral rim, liver abscess confirmed at right lobe of liver in 34 patients and at left lobe in 14 patients. Four patients had more than one abscess cavity. The cavity measured between 8 $\mathrm{cm}$ and $23 \mathrm{~cm}$ in diameter. Eighteen patients had diabetes mellitus (DM). Of the 48 patients, 9 had failed percutaneous drainage. Culture sensitivity of pus was done for all patients.

Written consent form was filled by every patient after detailed explanation of the surgery and possible complications.

\section{Patient Inclusion Criteria}

Patient having complex pyogenic liver abscess of more than $5 \mathrm{~cm}$ in diameter, multilocular that is not responding to percutaneous drainage, and/or antibiotics.

\section{Patient Exclusion Criteria}

Patients having small, solitary, and unilocular pyogenic liver abscess that responded to antibiotic treatment and/or percutaneous drainage were excluded.

\section{Imaging}

Imaging plays an important role in the diagnosis of liver abscess, and the main role of imaging is to detect early disease and confirm diagnosis. 6

Ultrasound and CT have high sensitivities for diagnosis of pyogenic liver abscess reaching to $97 \%$. By ultrasound small abscesses less than $2 \mathrm{~cm}$, appear as hypoechoic lesions or ill-defined areas of distorted parenchymal echogenicity within liver, large abscesses appears as hypoechoic or hyperechoic masses according to the presence of internal debris. Pyogenic abscess sometimes appears as solid lesion. ${ }^{7}$
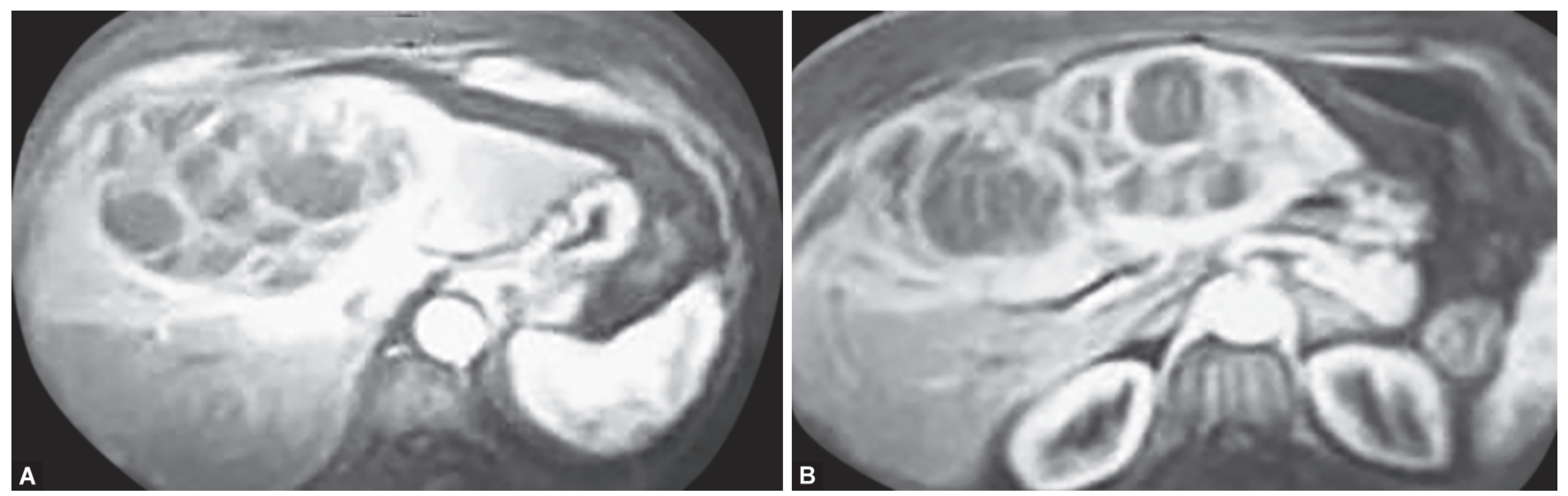

Figs $1 \mathrm{~A}$ and B: (A) MRI dynamic contrast enhancement study showing large bilobar multiloculated pyogenic liver abscesses with early enhancement of the inner layer with internal septal enhancement; (B) Delayed enhancement of the peripheral layer
The ability to differentiate an abscess from a neoplasm at nonenhanced ultrasound is limited compared with CT or MR imaging. However, if solid neoplasm starts to form necrosis, it could be differentiated from abscess by ultrasound. ${ }^{8}$

By contrast enhanced $C T$, pyogenic liver abscess appears as well-defined, low attenuation mass with an enhancing outer layer. It can appear as a single nonloculated cystic collection, multiloculated cystic mass, solid mass, or multifocal solid lesions. ${ }^{9}$

The characteristic imaging findings of abscess by contrast enhanced CT are called (double target sign) that is seen as central low attenuation cystic area surrounded by a high-density inner ring and a low-density outer ring. The inner layer shows early contrast enhancement with continuous enhancement at delayed phases. The outer layer appears of hypoattenuating with no enhancement in the early post contrast images then enhances in delayed phase. ${ }^{6}$

Another imaging findings called (cluster sign) that is seen with multiple small hypoattenuation abscesses aggregate and coalesce into one single large abscess cavity. Gas within lesions may be seen, either in the form of bubbles or appears as air-fluid leveling, which is a diagnostic sign for an abscess. ${ }^{10}$

At MR imaging, abscesses seen as central low T1 signal and high T2 signal intensity, but internal signal intensity may vary depending on the protein content. Pyogenic liver abscess appears by dynamic MRI contrast enhancement the same as in contrast enhanced CT, with early enhancement of the inner layers and internal septa and delayed enhancement of the peripheral layer ${ }^{6}$ (Fig. 1).

Some abscesses seen surrounded with edema signal, i.e., appears as bright T2 signal intensity with restricted diffusionweighted images and low signal intensity on ADC maps (Fig. 2). ${ }^{11}$

\section{Operative Techniques \\ Laparoscopic Drainage}

Under general anesthesia, initially pneumoperitoneum was created, then a $10 \mathrm{~mm}$ trochar was introduced, and laparoscope was inserted. Diagnostic laparoscopy was performed and then two 5 $\mathrm{mm}$ ports were introduced according to the location of the abscess. A $10 \mathrm{~mm}$ port was introduced for laparoscopic intraoperative ultrasound. The adhesions between the liver and bowel as well as the anterior abdominal wall were freed and the area where abscess present was exposed. Laparoscopic intraoperative ultrasound was done to detect the exact site and extent of the liver abscess, then deroofing of the abscess was done, and aspiration of the pus by the suction catheter and samples for pus culture was taken. The cavity 
was irrigated by normal saline, and proper hemostasis was secured. Finally, an abdominal drain was placed in the abscess cavity and another one in the pelvis (Fig. 3).

\section{Open Surgery}

A right subcostal incision or a midline abdominal incision was made according to abscess location. Intraoperative ultrasound was done to detect the exact site and extent of the liver abscess then de roofing of the abscess to drain pus and remove the fibrous septa. Hemostasis was secured and latex drainage tube was left (Fig. 2).

Operative and clinical data including operation time, intraoperative blood loss, postoperative complication rate, length of postoperative hospital stay, and rate of abscess recurrence were compared between the two groups.

Regular follow-up was done weekly for the first month after discharge then every 2 months for about one year. Clinical examination and abdominal ultrasound were done every visit.

\section{Study Design}

Combined retrospective and prospective study of all complex liver abscesses admitted to NHTMRI from January 2012 to January 2020 and comparison between laparoscopic and open surgical drainage as regards safety, efficacy, hospital stay, perioperative morbidity, mortality, and recurrence.



Fig. 2: Open surgical drainage of bilobar multiloculated pyogenic abscesses

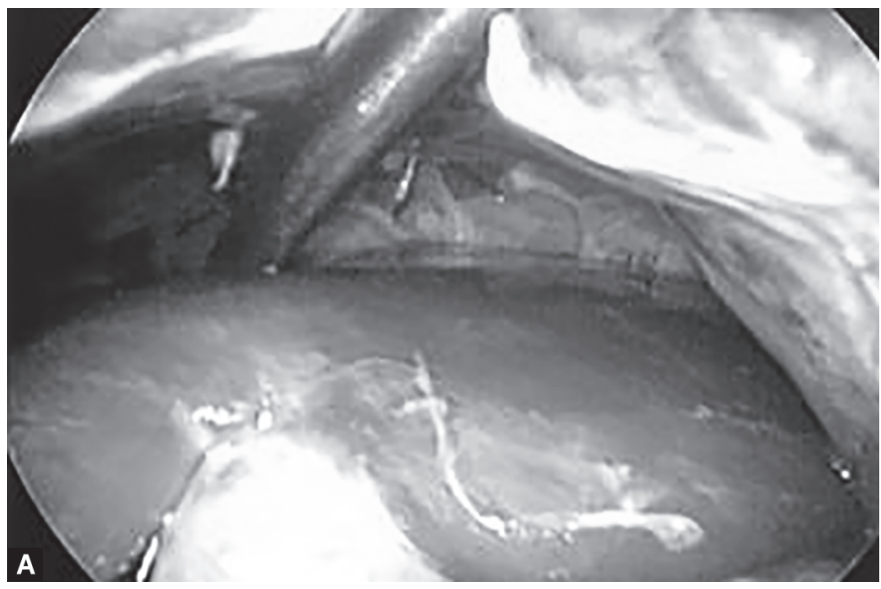

Figs $3 \mathrm{~A}$ and B: Laparoscopic drainage of liver abscesses

\section{Results}

Forty-eight patients ( 20 males and 28 females) with a median age of 54.5 years (ranges between 34 years and 65 years) were included in this study. The clinical and laboratory data of patients with CPLA at presentation are shown in Table 1.

All patient were diagnosed by one or two imaging modalities (ultrasonography, CT, or MRI) and all were successfully treated either by laparoscopic drainage or open surgery confirmed by at least one image modality, CT or MRI examination (Figs 4 and 5).

All patients received broad spectrum antibiotics. Nine patients had preoperative failed trial of percutaneous drainage. Twenty-six patients were managed by open surgical drainage and 22 patients by laparoscopic drainage. The operation time and hospital stay were less and oral feeding was started earlier in laparoscopic drainage group. Wound infection was higher in open drainage group. Abscess recurrence occurred once in laparoscopic group and once in open surgery group and both were successfully treated with percutaneous drainage. One laparoscopic operation was converted into open surgical drainage due to unsatisfactory laparoscopic drainage. Results are shown in Table 2.

In pus-culture study of the 48 patients, only 38 cases (79\%) had positive microbial reports while $21 \%$ had reports with no growth.

Table 1: Clinical and laboratory data of patients with complex pyogenic liver abscess at presentation

\begin{tabular}{lrl}
\hline Variables & LD group $(n=22)$ & OSD group $(n=26)$ \\
\hline Abdominal pain & 21 & 25 \\
Fever/rigors & 21 & 26 \\
Vomiting & 11 & 12 \\
Jaundice & 5 & 6 \\
Abdominal tenderness & 19 & 23 \\
Severe sepsis & 1 & 8 \\
Leukocytosis $(>11,000 / \mathrm{mL})$ & 22 & 26 \\
Elevated AST/ALT & 12 & 15 \\
Serum albumin $(<3.5 \mathrm{~g} / \mathrm{dL})$ & 7 & 9 \\
Total bilirubin $(>2 \mathrm{mg} / \mathrm{dL})$ & 6 & 9 \\
Serum creatinine & 1 & 8 \\
$(>1.4 \mathrm{mg} / \mathrm{dL})$ & &
\end{tabular}

$(>1.4 \mathrm{mg} / \mathrm{dL})$

LD, laparoscopic drainage; OSD, open surgical drainage

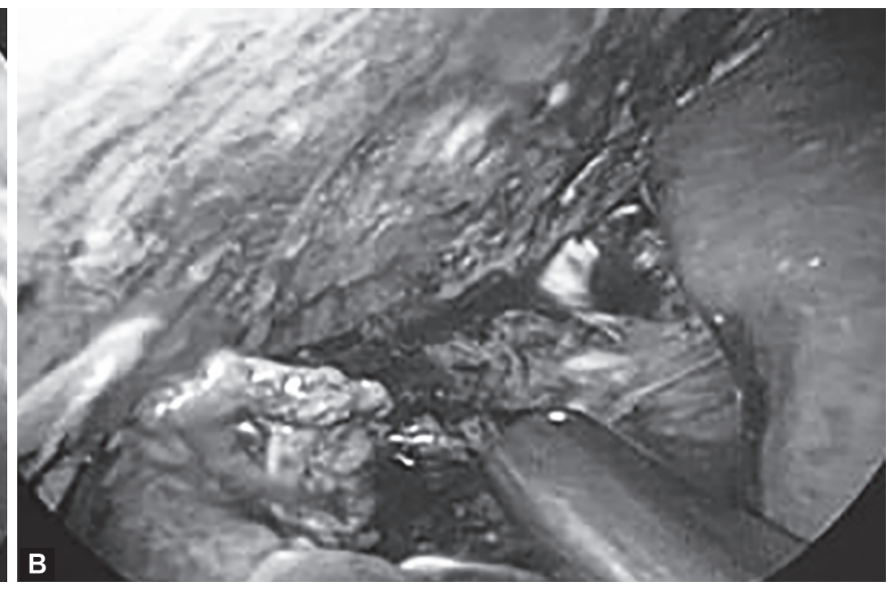



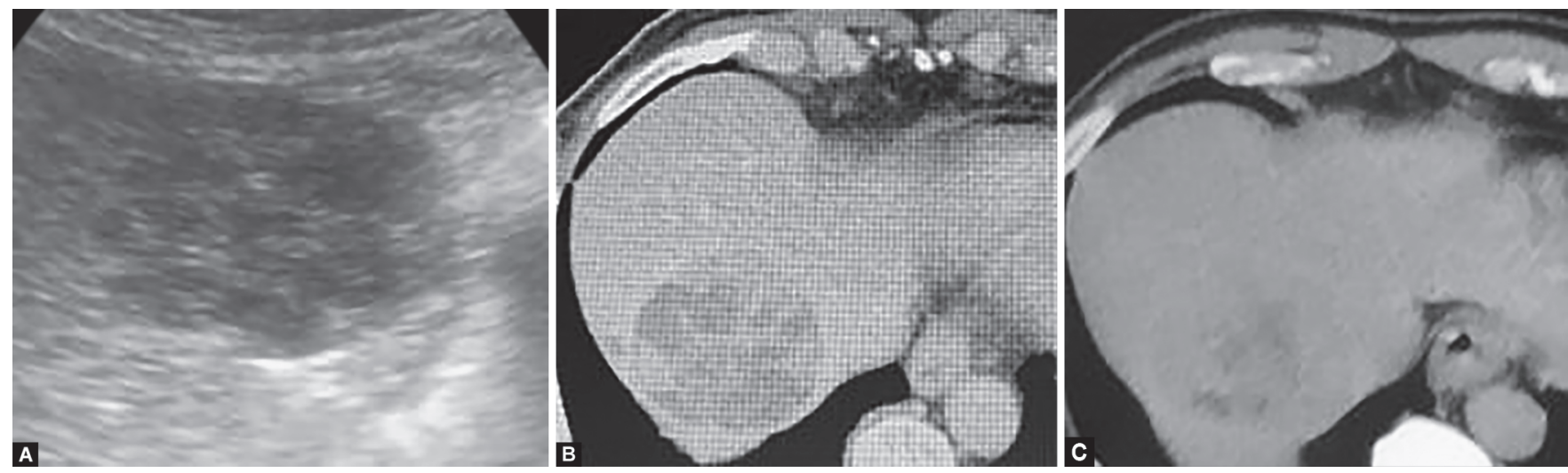

Figs 4A to C: (A) Ultrasonography of a case of right lobe pyogenic liver abscess of heterogeneous echogenic mass lesion before surgical interference (B) CT of the same case showing low attenuation mass with internal septa and debris; (C) CT done one week after surgery with nearly total resolution of the abscess
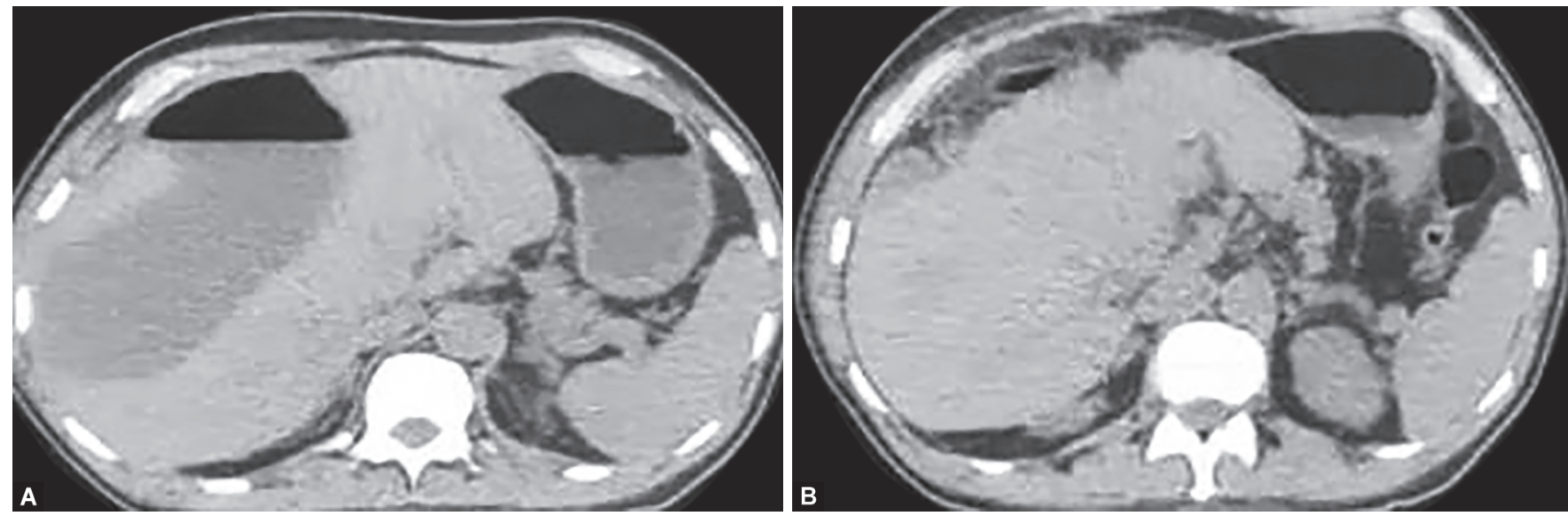

Figs 5A and B: (A) CT scan of a case before laparoscopic drainage showing large right lobe single nonloculated abscess at segment VIII with fluid leveling; (B) CT after laparoscopic drainage showing significance resolution of the lesion

The most common organisms identified were Escherichia coli $25 \%$ and Klebsiella pneumoniae $16 \%$, followed by anaerobics (12.5\%), Streptococcus spp (10.4\%), and polymicrobial (15\%).

\section{Discussion}

Before the 1970s, the mortality rate of PLA was high (more than $50 \%)$. With the development of imaging, surgical techniques, and effective broad-spectrum antibiotics, the mortality rate is markedly reduced. ${ }^{12,13}$ Complex pyogenic liver abscesses usually require surgical drainage either open surgical drainage (OSD) or laparoscopic drainage (LD) under cover of broad-spectrum antibiotics for effective management. ${ }^{4}$

This study conducted in NHTMRI which is a tertiary center specialized for liver surgery from January 2012 to January 2020. During this period, only 48 patients fulfilled criteria of complex liver abscess (more than $5 \mathrm{~cm}$ in diameter, multilocular). All patients received systemic antibiotics. Nine patients had preoperative failed trial of percutaneous drainage. Twenty-six patients were managed by open surgical drainage and 22 patients by laparoscopic drainage.

Eighteen patients (37.5\%) in the current study had DM which is comparable to study done by Li et al. in 2018 on 246 PLA patients with $90(36.6 \%)$ of them had diabetes and higher than the study of Serraino et al. in which 25 patients of 109 (23\%) had DM. ${ }^{14}$
Table 2: Results of laparoscopic drainage group and open surgical drainage group

\begin{tabular}{|c|c|c|}
\hline Variables & LD group $(n=22)$ & OSD group $(n=26)$ \\
\hline $\begin{array}{l}\text { Operative time (median } \\
\text { and range), minutes }\end{array}$ & $86(75-125)$ minutes & $\begin{array}{l}105 \\
(95-140) \text { minutes }\end{array}$ \\
\hline $\begin{array}{l}\text { Hospital stay (median } \\
\text { and range), days }\end{array}$ & 5 (4-7) day & $8(6-11)$ day \\
\hline $\begin{array}{l}\text { Comorbidity }(18 / 48 \\
\text { diabetics: patients } \\
n \text { and \%) }\end{array}$ & $12 / 22(54.5 \%)$ & $6 / 26(23 \%)$ \\
\hline $\begin{array}{l}\text { Postoperative wound } \\
\text { infection ( } n \text { and \%) }\end{array}$ & $0(0 \%)$ & $2 / 26(7.7 \%)$ \\
\hline Perioperative mortality & $0(0 \%)$ & $0(0 \%)$ \\
\hline $\begin{array}{l}\text { Failed preoperative } \\
\text { percutaneous drainage } \\
\text { ( } 9 / 48 \text { patients) }\end{array}$ & $2 / 22(9 \%)$ & $7 / 26(27 \%)$ \\
\hline Abscess recurrence & $1 / 22(4.5 \%)$ & $1 / 26(3.8 \%)$ \\
\hline
\end{tabular}

$\mathrm{LD}$, laparoscopic drainage; OSD, open surgical drainage

In our study, the four patients that had more than one abscess cavity were diabetic and the two patients who had recurrence were also diabetic. 
Preoperative ultrasound, CT, MRI, and intraoperative laparoscopic ultrasound help to identify abscess localization, liquefaction, cavity size, pus volume, multiloculation, septa, and abscess number. Abdominal ultrasonography was done in all patients and was diagnostic alone in 24 (50\%) of cases; however, $\mathrm{CT}$ was required in 22 patients and MRI in 2 patients to confirm the diagnosis. This is comparable to Serraino et al. study in which ultrasound was diagnostic in $42.4 \%$, CT scan in $51.1 \%$, and MRI in $3.3 \%$ of their cases. ${ }^{14}$

Seventy-nine percentage of our patients in this study had positive culture reports while $21 \%$ had reports with no growth. The most common organisms identified were Escherichia coli $25 \%$ and Klebsiella pneumoniae $16 \%$. This is comparable to results of Malik et al., Serraino et al., and different from Liu et al. who found that 25 positive results of 66 cases (37.9\%), with Klebsiella pneumoniae detected in 15 cases $(60.0 \%)$ as the most common pathogen. ${ }^{14-16}$

The operative time and hospital stay were less and oral feeding was started earlier in laparoscopic drainage group. This is comparable to Tu et al. study as regards hospital stay and oral feeding but not for operation time as Tu and his colleagues had longer LS time perhaps because they managed the biliary pathology at the same time. ${ }^{17}$

In the current study, there were no perioperative mortality which is comparable to Tu et al. study and in contrary to the study done by Malik et al., in which mortality occurred in 19 of 169 patients with pyogenic liver abscesses and it was higher in the nonsurgical drainage group (7 out of 42 patients $16.6 \%$ ) than the surgically drained group (12 out of 127 patients $9.4 \%$ ). ${ }^{15,17}$ As regards the recurrence rate, it occurred in two cases (4.2\%), one case in laparoscopic group 1/22 (4.5\%) and one case in open group $1 / 26(3.8 \%)$ and both were successfully treated with percutaneous drainage.

\section{Conclusion}

Both laparoscopic and open surgical drainage of PLA are safe and effective. Laparoscopic drainage has less operative time, morbidity, and hospital stay; however, open drainage is considered the management of choice for patients with critical condition or with failed percutaneous drainage. When laparoscopic drainage is unsatisfactory conversion to open surgical drainage is recommended.

\section{Disclosure}

Informed written consent was obtained from the patients.

\section{References}

1. Chung YF, Tan YM, Lui HF, et al. Management of pyogenic liver abscesses-percutaneous or open drainage? Singapore Med J 2007;48(12):1158-1165.

2. Wankg W, Lee WJ, Wei PL, et al. Laparoscopic drainage of pyogenic liver abscesses. Surg Today 2004;34(4):323-325. DOI: 10.1007/s00595003-2709-x.

3. Cioffi L, Belli A, Limongelli $P$, et al. Laparoscopic drainage as first line treatment for complex pyogenic liver abscesses. Hepatogastroenterology 2014;61(131):771-775.

4. Bowers ED, Robison DJ, Doberneck RC. Pyogenic liver abscess. World J Surg 1990;14(1):128-132. DOI: 10.1007/BF01670563.

5. Aydin C, Piskin T, Sumer F, et al. Laparoscopic drainage of pyogenic liver abscess. JSLS 2010;14(3):418-420. DOI: 10.4293/108680810X129 24466006567.

6. Bächler P, Baladron MJ, Menias C, et al. Multimodality imaging of liver infections: differential diagnosis and potential pitfalls. Radiographics 2016;36(4):1001-1023. DOI: 10.1148/rg.2016150196.

7. Lin AC, Yeh DY, Hsu YH, et al. Diagnosis of pyogenic liver abscess by abdominal ultrasonography in the emergency department. Emerg Med J 2009;26(4):273-275. DOI: 10.1136/emj.2007.049254.

8. Benedetti NJ, Desser TS, Jeffrey RB. Imaging of hepatic infections. Ultrasound Q 2008;24(4):267-278. DOI: 10.1097/ RUQ.0b013e31818e5981.

9. Mortelé KJ, Segatto E, Ros PR. The infected liver: radiologic-pathologic correlation. Radiographics 2004;24(4):937-955. DOI: 10.1148/ rg. 244035719.

10. Alsaif HS, Venkatesh SK, Chan DS, et al. CT appearance of pyogenic liver abscesses caused by Klebsiella pneumoniae. Radiology 2011;260(1):129-138. DOI: 10.1148/radiol.11101876.

11. Doyle DJ, Hanbidge AE, O'Malley ME. Imaging of hepatic infections. Clin Radiol 2006;61(9):737-748. DOI: 10.1016/j.crad.2006.03.010.

12. Huang CJ, Pitt HA, Lipsett PA, et al. Pyogenic hepatic abscess. Changing trends over 42 years. Ann Surg 1996;223(5):600-607. DOI: 10.1097/00000658-199605000-00016.

13. Liu CH, Gervais DA, Hahn PF, et al. Percutaneous hepatic abscess drainage: do multiple abscesses or multiloculated abscesses preclude drainage or affect outcome? J Vasc Interv Radiol 2009;20(8):10591065. DOI: 10.1016/j.jvir.2009.04.062.

14. Serraino C, Elia C, Bracco C, et al. Characteristics and management of pyogenic liver abscess: a European experience. Medicine (Baltimore) 2018;97(19):e0628. DOI: 10.1097/MD.0000000000010628.

15. Malik AA, Bari SU, Rouf KA, et al. Pyogenic liver abscess: changing patterns in approach. World J Gastrointest Surg 2010;2(12):395-401. DOI: 10.4240/wjgs.v2.i12.395.

16. Liu L, Chen W, LuX, et al. Pyogenic liver abscess: a retrospective study of 105 cases in an emergency department from East China. J Emerg Med 2017;52(4):409-416. DOI: 10.1016/j.jemermed.2016.09.026.

17. Tu JF, Huang XF, Hu RY, et al. Comparison of laparoscopic and open surgery for pyogenic liver abscess with biliary pathology. World J Gastroenterol 2011;17(38):4339-4343. DOI: 10.3748/wjg.v17.i38.4339. 